\title{
Erratum to: Brown root rot caused by Phellinus noxius in the Ogasawara (Bonin) islands, southern Japan - current status of the disease and its host plants
}

\author{
Norio Sahashi $^{1} \cdot$ Mitsuteru Akiba $^{1} \cdot$ Yuko Ota $^{1} \cdot$ Hayato Masuya $^{2} \cdot$ Tsutomu Hattori $^{1}$. \\ Akitsugu Mukai ${ }^{3} \cdot$ Ritsuko Shimada $^{3}$ - Tsuyoshi Ono ${ }^{4}$ - Toyozo Sato ${ }^{5}$
}

Published online: 23 November 2015

(C) Australasian Plant Pathology Society Inc. 2015

\section{Erratum to: Australasian Plant Dis. Notes} DOI 10.1007/s13314-015-0183-0

The original version of this article contained a spelling mistake in the title and some incorrect accession numbers in paragraph 3. The changes are underlined in the following corrected text.

The online version of the original article can be found at http://dx.doi.org/ 10.1007/s13314-015-0183-0.

Norio Sahashi

sahasi@affrc.go.jp

1 Department of Forest Microbiology, Forestry and Forest Products Research Institute (FFPRI), Matsunosato 1, Tsukuba 305-8687, Japan

2 Tohoku Research Center, FFPRI, Morioka 020-0123, Japan

3 Greenery Environment Section, Ogasawara Branch of Tokyo Metropolitan Government, Aza-Nishimachi, Chichijima 100-2101, Japan

4 Agro-Environment Division, Tokyo Metropolitan Agriculture and Forestry Research Center, Fujimi-cho 3-8-1,

Tachikawa, Tokyo 190-0013, Japan

5 Genetic Resources Center, National Institute of Agrobiological Sciences, (NIAS), Kannonndai 2-1-2, Tsukuba 305-8602, Japan

\section{Article Title:}

Brown root rot caused by Phellinus noxius in the Ogasawara (Bonin) islands, southern Japan - current status of the disease and its host plants

$12^{\text {th }}$ sentence under paragraph 3 :

For molecular identification, we used two LSU data (DDBJ Accession Nos. LC049950 and LC049951) derived from two P. noxius isolates (WD 1223 obtained from a fruiting body and FFPRI 411141 obtained from a single basidiospore) as reference sequences.

$14^{\text {th }}$ sentence under paragraph 3 :

Then, representative isolates including FFPRI 411141 collected from each of 38 different tree species were chosen (Table 1), and the DNA sequences of the LSU of the isolates were compared with the $P$. noxius reference sequences described above. $15^{\text {th }}$ sentence under paragraph 3 :

Isolate FFPRI 411141 was treated as a representative isolate because this was the only isolate obtained from Melia azedarach var. subtripinnata.

$16^{\text {th }}$ sentence under paragraph 3:

These isolates were deposited in FFPRI Genebank (FFPRI 411120-411156) and NAIS Genebank (MAFF243592) (Table 1). $17^{\text {th }}$ sentence under paragraph 3 :

All sequence data determined in the present study were also deposited in the DDBJ (Accession Nos. LC066600-066636). 